\title{
La historia natural de la sensibilización fúngica en el asma
}

\section{The natural history of fungal sensitization in asthma}

\author{
LUisa Fernanda ManRiQue, MD., MSc.(1); Claudia Patricia Zuluaga, MD.(2)
}

\section{Resumen}

El asma severa con sensibilización fúngica forma parte del continuo de enfermedad, que inicia con el asma y finaliza con la aspergilosis broncopulmonar alérgica. Este grupo de entidades comúnmente son subdiagnosticadas y se pueden asociar a daños pulmonares irreversibles como bronquiectasias y fibrosis pulmonar. Recientemente se han descrito nuevos criterios diagnósticos que ayudarán a un manejo más temprano y beneficiarán la investigación clínica.

Palabras clave: asma, Aspergillus, aspergilosis broncopulmonar alérgica, ABPA, asma severa con sensibilización fúngica.

\begin{abstract}
Severe asthma with fungal sensitization (SAFS) is part of the continuum of diseases that begins with asthma and ends with allergic bronchopulmonary aspergillosis. These conditions often run underdiagnosed, and can be associated with irreversible pulmonary damage such as bronchiectasis and pulmonary fibrosis. New diagnostic criteria have been recently described, which are expected to aid in earlier management and to benefit clinical investigation.
\end{abstract}

Keywords: asthma, Aspergillus, allergic bronchopulmonary aspergillosis, severe asthma with fungal sensitization, ABPA.

\section{Introducción}

La relación entre asma y exposición fúngica no es nueva, pero la descripción de nuevos fenotipos que representan un continuo en la evolución de la enfermedad, es reciente. Este continuo abarca inicialmente el asma y finaliza con la aspergilosis broncopulmonar alérgica (ABPA). Dentro de este espectro de manifestaciones se encuentra el asma severa con sensibilización fúngica (SAFS) (1), el cual se presenta luego de la exposición a Aspergillus $\mathrm{u}$ otros hongos, y produce una cascada inflamatoria en la que predomina la respuesta de las células Thelper 2 (TH2) y finaliza, de acuerdo con la susceptibilidad genética del individuo, en ABPA con cambios irreversibles como bronquiectasias y fibrosis $(1,2,5)$.

El asma severa con sensibilización fúngica es un diagnóstico de exclusión y se basa en la presencia de asma severa, sensibilización fúngica y ausencia de ABPA (1). Allí radica la dificultad en el diagnóstico, ya que los criterios diagnósticos de ABPA son variables y contradictorios, lo que posiblemente
(1)Internista, Neumóloga, Hospital General de Medellín y CEMDE S.A, Medellín, Colombia.

(2)Radióloga, Hospital Militar de Medellín, Hernán Ocazionez y Cia S.A, Clínica Medellín de Occidente, Medellín, Colombia. Correspondencia: Luisa Fernanda Manrique, correo electrónico: luisa40co@gmail. com

Recibido: 18/05/15. Aceptado: 28/05/15. 
conduce al subdiagnóstico de la enfermedad. Se plantean por tanto nuevos criterios diagnósticos por parte de la ISHAM (del inglés International Society for Human and Animal Micology) para lograr un diagnóstico más temprano y así minimizar las complicaciones relacionadas con la enfermedad (2).

Se expone el caso de un paciente con asma severa con sensibilización fúngica con criterios incompletos para ABPA.

\section{Caso}

Hombre de 54 años con diagnóstico de asma alérgica severa, antecedentes personales de pansinusitis crónica, migraña y tabaquismo pasivo, conocido en la consulta externa de Neumología desde el año 2015, con historia de tres exacerbaciones asmáticas durante el 2014, uso de esteroides sistémicos y persistencia de síntomas respiratorios. Las pruebas de función pulmonar mostraron obstrucción moderada con hiperreactividad, atrapamiento e hiperinsuflación y aumento de resistencias. En los exámenes de laboratorio se evidenció eosinofilia en sangre (1.670 células $/ \mu 1$ ) pero no en esputo, e inmunoglobulina $\mathrm{E}$ total elevada $(554 \mathrm{UI} / \mathrm{ml})$, (posiblemente subestimada por el uso de esteroides sistémicos).

La radiografía de tórax fue normal y el prick test demostró sensibilización para hongos (Alternaria alternata, Cladosporium herbarum y Trichophyton mentagrophytes). Al manejo previamente establecido de esteroide combinado con beta 2 de larga acción, antileucotrienos, esteroide nasal, vacunación para influenza y neumococo y rehabilitación pulmonar se adicionó omalizumab, evidenciándose marcada mejoría clínica y del ACT (Asthma Control Test), menor variabilidad en la medición del pico flujo espiratorio (PEF) y menor uso de recursos de salud.

Ocho meses después de iniciado el manejo se evidenció deterioro clínico y espirométrico con disminución de ACT y de PEF y múltiples consultas al servicio de urgencias. Ante la evidencia de deficiencia de vitamina $\mathrm{D}(14,59 \mathrm{ng} / \mathrm{ml})$ se inició reposición y se adicionó manejo anticolinérgico de larga acción. En la tomografía de tórax se observaron bronquiolectasias y engrosamientos de paredes bronquiales (figura 1). Ante estos hallazgos se sospechó aspergilosis versus micosis broncopulmonar alérgica y se repitieron las pruebas cutáneas prick test con evidencia de sensibilización a Aspergillus fumigatus, IgE específica positiva para Aspergillus fumigatus y para Trichophyton y una IgG negativa para Aspergillus fumigatus. Con estos hallazgos se consideró asma severa con sensibilización fúngica con criterios incompletos para ABPA pero que representa un paciente "en riesgo para ABPA" y ante el deterioro clínico se inició prednisona e itraconazol por 16 semanas, con buena respuesta clínica.
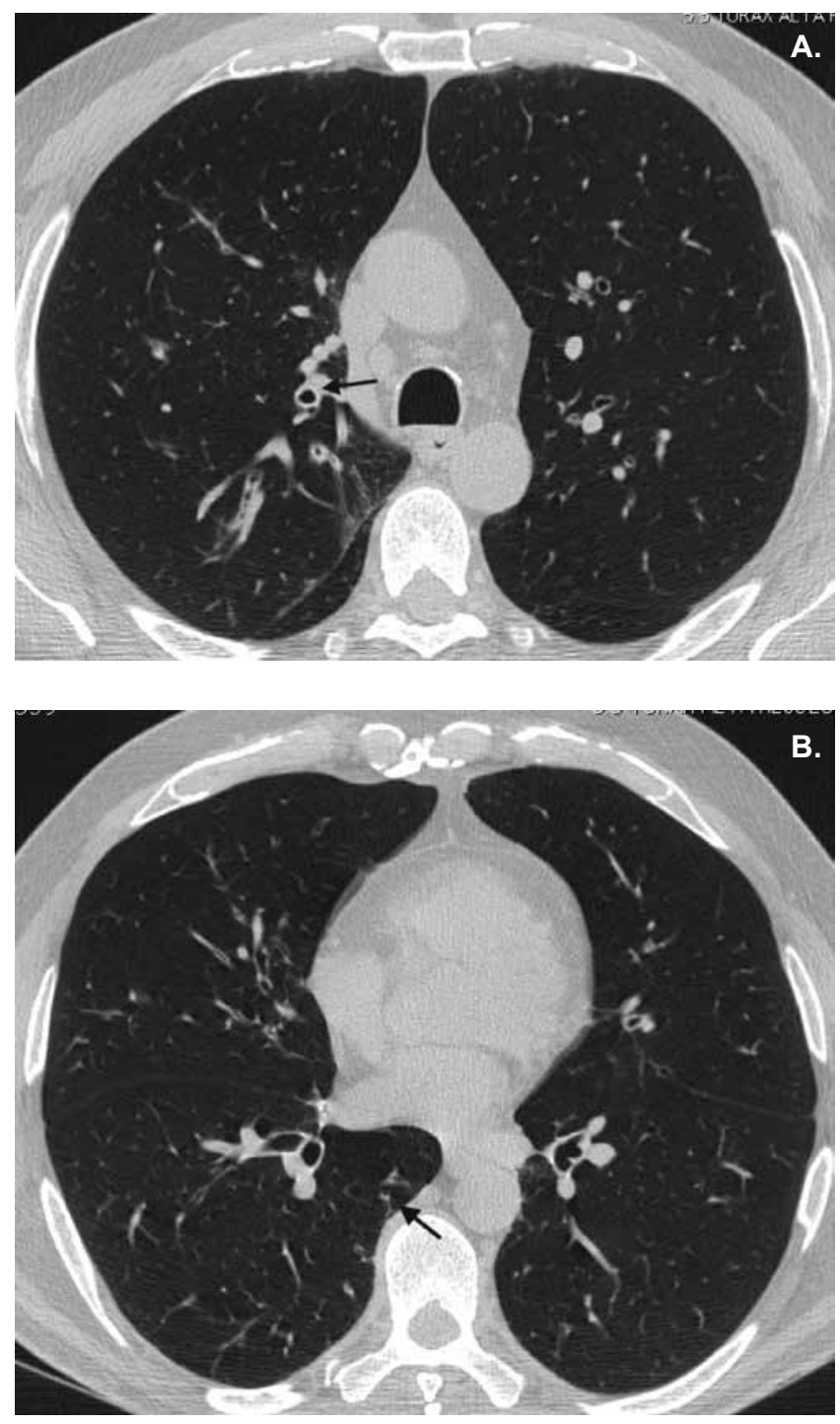

Figura 1. Tomografía de tórax; corte axial. A: Engrosamiento de paredes bronquiales. B: Escasas bronquiolectasias. 
En la tabla 1 se resumen los datos de los paraclínicos del paciente.

\section{Discusión}

La prevalencia para SAFS y ABPA se estima en 28 y $12,9 \%$ respectivamente en clínicas de asma (3). Varias especies de hongos han sido descritas en casos de SAFS y ABPA, y es posible que una prevalencia más alta de sensibilización a varios alergenos fúngicos se relacione con mayor uso de recursos de salud (2).
Aun no es claro si la relación entre asma severa y sensibilización fúngica es una asociación causal directa o indirecta, pero se cree que esa sensibilización es un continuo que lleva a aspergilosis broncopulmonar alérgica, posiblemente por mayor respuesta inflamatoria en ABPA mediada por TH2 (1, 4). En pacientes con predisposición genética (polimorfismos HLA) la exposición fúngica produce colonización, liberación de antígenos y proteasas que aumentan la respuesta de las células TH2 y la

Tabla 1. Datos de laboratorio y función pulmonar.

\begin{tabular}{|c|c|c|c|}
\hline Variable & Rangos de referencia & 2015 & 2016 \\
\hline \multicolumn{4}{|l|}{ Espirometría } \\
\hline VEF $_{1}$ litros prebroncodilatador (post) & - & $2,30(2,61)$ & $1,14(1,80)$ \\
\hline $\mathrm{VEF}_{1} \%$ predicho prebroncodilatador (post) & - & $69(79)$ & $34(54)$ \\
\hline \multicolumn{4}{|l|}{ Caminata de 6 minutos } \\
\hline Metros caminados & - & 533 & 460 \\
\hline$\%$ predicho & - & 96 & 69 \\
\hline Cuestionario respiratorio St. George puntaje total & $0-100$ & 57,4 & - \\
\hline Inmunoglobulina $E$ total $(\mathrm{Ul} / \mathrm{ml})$ & $0-100$ & 554 & - \\
\hline \multicolumn{4}{|l|}{ Prick test (mm) } \\
\hline Alternaria alternata & - & 4 & NA \\
\hline Aspergillus fumigatus & - & neg & 6 \\
\hline Asperglillus niger & - & neg & NA \\
\hline Trichophyton mentagrophytes & - & 3 & NA \\
\hline Cladosporium herbarum & - & 4 & NA \\
\hline Eosinófilos en esputo & - & - & neg \\
\hline Eosinófilos en sangre (no absoluto) & $0-700$ & 1.670 & 880 \\
\hline Vitamina D 25 hidroxi (ng/ml) & $30-100$ & - & 14,59 \\
\hline \multicolumn{4}{|l|}{ Inmunoglobulina E específica (kU/L) } \\
\hline Alternaria alternata & nivel muy bajo $0,10-0,49$ & - & 0,34 \\
\hline Trichophyton mentagrophytes & alto clase $3: 3,5-17,49$ & - & 7,88 \\
\hline Aspergillus fumigatus & bajo clase 1: 0,35 - 0,69 & - & 0,43 \\
\hline Cladosporium herbarum & negativo $<0,35$ & - & $<0,35$ \\
\hline Inmunoglobulina G Aspergillus fumigatus (mg/L) & $0-102$ & - & 14,2 \\
\hline
\end{tabular}

neg: negativo, NA: no aplica 
liberación subsecuente de interleucinas (IL) como IL-4, IL-5 e IL-13 promoviendo la síntesis de IgE e IgG. Esto a su vez estimula la proliferación de mastocitos y eosinófilos en el epitelio bronquial, que con el tiempo causa destrucción, remodelación, bronquiectasias y fibrosis $(2,5)$.

Esta respuesta de las células $\mathrm{T}$ está restringida por HLA DR2 o HLA DR5 $(2,5)$. Los alergenos de Aspergillus fumigatus evocan diferentes respuestas inmunológicas que determinan las manifestaciones de la enfermedad; tipo 1 (hipersensibilidad inmediata) tipo 3 (complejos antígeno-anticuerpo) y tipo IVb (respuesta celular inflamatoria mediada por eosinófilos) (5).

Las manifestaciones clínicas en SAFS son similares a las observadas en el asma severa. En general, los pacientes con ABPA cursan con asma no controlada, pero hasta el $19 \%$ pueden ser asintomáticos (2). Los síntomas más frecuentes son sibilancias, hemoptisis, tos productiva, fiebre, malestar general, pérdida de peso y fatiga (2-6).

Las pruebas cutáneas de hipersensibilidad inmediata son la base del diagnóstico tanto en ABPA como en SAFS, y representan la presencia de IgE específico para A. fumigatus. Se recomienda inicialmente realizar el prick test, y si es este es negativo elegir la prueba intradérmica $(1,2,6)$.

La inmunoglobulina $\mathrm{E}$ total es útil para el diagnóstico como para el seguimiento del ABPA, pero también permite diferenciar SAFS de ABPA (figura 2) $(1,2)$. Su interpretación no es sencilla; puede verse afectada con el uso de medicamentos como el omalizumab y los esteroides sistémicos y su valor de corte ha sido variable en los diferentes algoritmos propuestos $(2,16)$. Ciertos grupos proponen el valor de $1.000 \mathrm{mg} / \mathrm{ml}(417 \mathrm{UI} / \mathrm{ml})$ y otros 1.000 $\mathrm{UI} / \mathrm{ml}(14,15)$. No existen curvas de característica operativa del receptor (ROC) que analicen el nivel de IgE en ABPA, SAFS y asma con sensibilización fúngica (2). El grupo de expertos de la ISHAM propone el valor de $1.000 \mathrm{UI} / \mathrm{ml}$ como punto de corte, realizado temprano en el curso de la enfermedad cuando no se han usado esteroides sistémicos (2).
Los anticuerpos IgE específicos para Aspergillus fumigatus son un hallazgo característico del ABPA y SAFS. Se ha propuesto un valor de corte de más del doble de las muestras combinadas de suero de los pacientes sensibilizados para Aspergillus, pero como esto rara vez es posible, se plantea el valor $>0,35 \mathrm{kUA} / \mathrm{L}(1,2)$.

La eosinofilia periférica es un criterio poco específico, es alta en otras enfermedades y puede disminuir rápidamente con el uso de esteroides sistémicos. Ante la evidencia de que solo el $40 \%$ de los pacientes con ABPA presentan eosinofilia mayor de 1.000 células/U1 se plantea un punto de corte más bajo (500 células/U1) $(2,17)$.

La IgG para Aspergillus fumigatus está presente en el 69 al 90\% de los pacientes con ABPA, no es especifica para ABPA y es positiva en la aspergilosis pulmonar crónica (2); así mismo, se ha descrito que es negativa en pacientes con SAFS (7). El cultivo de esputo para Aspergillus fumigatus no es diagnóstico para ABPA pero puede tener utilidad antes del inicio de la terapia antifúngica (2).

Los pacientes con asma severa y sensibilización fúngica presentan diversas alteraciones parenquimatosas. La radiografía de tórax no tiene buena sensibilidad para detectar bronquiectasias y puede ser normal hasta en el $50 \%$ de los pacientes con ABPA (8). Los hallazgos en esta modalidad de imagen son opacidades lineales paralelas y en anillo (bronquiectasias) y opacidades alveolares (consolidación); estas últimas son menos frecuentes en las nuevas series porque con el advenimiento de la tomografía computarizada de tórax de alta resolución (TCAR) se encontró que la mayoría de las consolidaciones visualizadas en la radiografía correspondían a bronquiectasias impactadas (8).

La TCAR tiene una sensibilidad y especificidad para el diagnóstico de bronquiectasias del $96-98 \%$ y $93-99 \%$ respectivamente, en tanto que en la serie de Agarwal et al. fue normal en 22 de 60 pacientes con ABPA (36\%) (8). En la serie de Menzies et al. los principales hallazgos escanográficos fueron engrosamiento de paredes bronquiales 


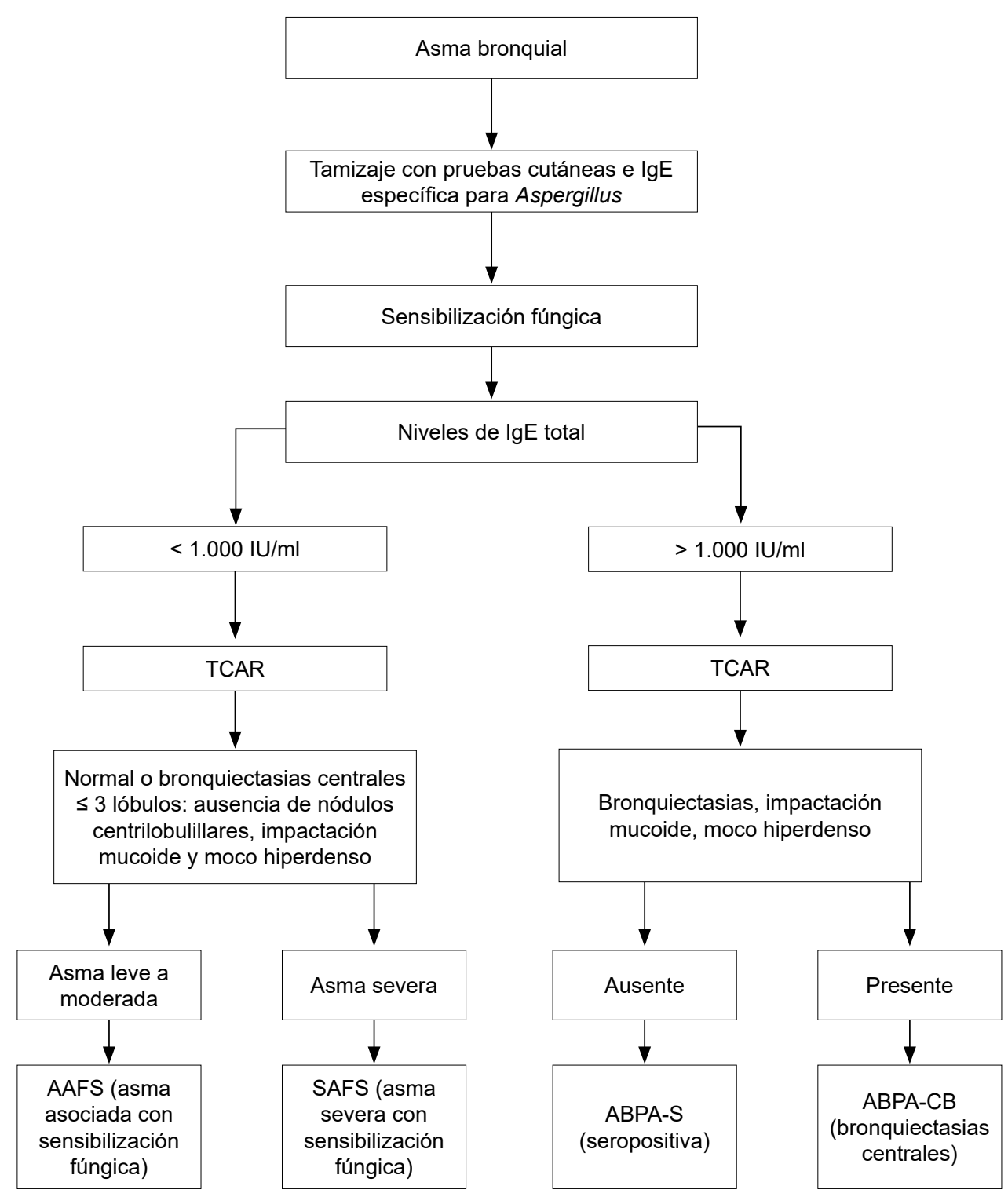

Figura 2. Algoritmo diagnóstico para el asma relacionada con sensibilización fúngica.

TCAR: Tomografía computarizada de tórax de alta resolución. Tomado y adaptado de: Agarwal R. Severe asthma with fungal sensitization. Curr Allergy Asthma Resp. 2011;11:403-13.

$(41,3 \%)$, bronquiectasias $(35,3 \%)$, atrapamiento aéreo $(20,3 \%)$ y dilatación bronquial $(16,3 \%)(8,9)$. Se ha propuesto una nueva clasificación radiológica para la ABPA basada en hallazgos tomográficos (2) (tabla 2).

Las bronquiectasias se distribuyen con mayor frecuencia en los lóbulos superiores y son de localización central (dos tercios mediales de los campos pulmonares); sin embargo hasta el $40 \%$ de estas se extienden a la periferia, de acuerdo con la serie de Agarwal et al., con prevalencia del 11 y $61 \%$ en otras series (2).

La impactación mucosa ocurre como consecuencia de la disfunción ciliar y la excesiva producción de moco. Presenta valores de atenuación altos en la tomografía (mayor densidad que la musculatura paravertebral) debido a la presencia de sales de calcio, hierro y magnesio y se observa hasta en el 30\% de los pacientes 
Tabla 2. Clasificación de la ABPA basada en CT.

\begin{tabular}{|ll|}
\hline Clasificación & \multicolumn{1}{c|}{ Características } \\
ABPA - S & $\begin{array}{l}\text { Todos los criterios diagnósticos para } \\
\text { ABPA, sin alteraciones en el TCAR }\end{array}$ \\
& $\begin{array}{l}\text { Todos los criterios diagnósticos para } \\
\text { ABPA, incluyendo bronquiectasias en } \\
\text { la TCAR }\end{array}$ \\
ABPA - MHD & $\begin{array}{l}\text { Todos los criterios diagnósticos para } \\
\text { ABPA, incluyendo moco hiperdenso }\end{array}$ \\
& $\begin{array}{l}\text { Al menos dos de tres características } \\
\text { dadas por: fibrosis pulmonar, } \\
\text { cicatriz parenquimatosa, lesiones } \\
\text { fibrocavitarias, aspergiloma y } \\
\text { engrosamiento pleural con ausencia } \\
\text { de impactación de moco con } \\
\text { hiperdensidad }\end{array}$ \\
\hline
\end{tabular}

ABPA - S: aspergilosis broncopulmonar alérgica seropositiva, ABPA $B$ : aspergilosis broncopulmonar alérgica con bronquiectasias, ABPA - MHD: aspergilosis broncopulmonar alérgica con moco hiperdenso, ABPA - FCPP: aspergilosis broncopulmonar alérgica con fibrosis pleuropulmonar crónica, TCAR: tomografía computarizada de tórax de alta resolución, ABPA: aspergilosis broncopulmonar alérgica. 19. Tomado y adaptado de: Agarwal R, Chakrabarti A, Shah A, Gupta D, et al. Allergic broncopulmonary aspergilosis: review of the literature and proposal of new diagnostic and classification criteria. Clin Exp Allerg. 2013;43:850-73.

(9-11). El hallazgo de impactación mucosa con hiperdensidad se considera específico para el diagnóstico de ABPA (10). Los tapones de moco impactado contienen Aspergilus y eosinófilos que pueden dar la apariencia de imágenes en dedo de guante (11).

Se han descrito otros hallazgos en los pacientes con ABPA; en la serie de Angus et al. se encontró engrosamiento de las paredes bronquiales (94\%), engrosamiento pleural y opacidades parenquimatosas $(82 \%)$, atelectasia/pérdida de volumen y enfisema $(52 \%)(12)$. Las opacidades transitorias se visualizan generalmente durante la exacerbación aguda de la enfermedad (8). Las manifestaciones radiológicas inusuales de la ABPA son opacidades nodulares de distribución miliar, líquido pleural, masas pulmonares y opacidades parahiliares que simulan adenopatías (2).

Los criterios diagnósticos más usados han sido los de Rosenberg-Patterson (13), pero con múltiples limitaciones. No existe una definición clara del número de criterios mayores y menores necesarios, las bronquiectasias representan una complicación de la enfermedad no una manifestación inicial, la especificidad de la IgG para Aspergillus en ABPA no es adecuada y no existe validación de los puntos de corte usados para los niveles de IgE total y eosinófilos (2). La ISHAM propone una clasificación más simple que permita un diagnóstico más temprano (tabla 3). Posiblemente muchos pacientes en la práctica clínica no cumplirán todos los criterios y se propone una categoría "en riesgo para ABPA" que obliga a una monitorización y a un seguimiento más estrecho (2).

El tratamiento para SAFS es igual al manejo descrito para el asma severa (1). Existe evidencia que el omalizumab puede ser beneficioso en ABPA y podría esperarse que también lo sea para SAFS,

Tabla 3. Criterios diagnósticos para ABPA propuestos por la ISHAM.

\begin{tabular}{|l|}
\hline \multicolumn{1}{c|}{ Criterios diagnósticos } \\
Condiciones que predisponen \\
Asma bronquial, fibrosis quística \\
Criterios obligatorios (ambos deben estar presentes) \\
Pruebas cutáneas de hipersensibilidad tipo I positiva \\
para Aspergillus o IgE específico para Aspergillus \\
fumigatus positivo \\
IgE total elevada (>1.000 IU/ml)* \\
Otros criterios (al menos dos de tres) \\
Presencia de precipitinas o anticuerpos IgG para \\
Aspergillus fumigatus en suero \\
Hallazgos radiográficos consistentes con ABPA ${ }^{\circ}$ \\
Conteo de eosinófilos $>500$ células/ $\mu l$ (sin ingesta de \\
esteroides, puede ser un dato histórico)
\end{tabular}

* Si el paciente cumple todos los otros criterios, un valor de IgE total $<1.000 \mathrm{IU} / \mathrm{ml}$ puede ser aceptable.

'Los hallazgos radiográficos puede ser transitorios (p. ej. consolidación, nódulos, opacidades en dedo de guante, opacidades en carrilera) o permanentes ( $p$. ej. líneas paralelas y en anillo, bronquiectasias y fibrosis pleuropulmonar). ABPA: aspergilosis broncopulmonar alérgica.

Tomado y adaptado de: Agarwal R, Chakrabarti A, Shah A, Gupta D, et al. Allergic broncopulmonary aspergilosis: review of the literature and proposal of new diagnostic and classification criteria. Clin Exp Allerg. 2013;43:850-73. 
no obstante, esto requiere confirmación ya que la mayoría de los estudios realizados son reportes de casos y pequeñas series (18). El tratamiento para ABPA busca controlar el asma, prevenir las exacerbaciones y evitar la aparición de bronquiectasias (2). Se basa en el uso de esteroides sistémicos e itraconazol; sin embargo, cabe resaltar que los regímenes descritos en cuanto a duración y dosis, aun no son claros (2), y que existen pocos estudios acerca del uso de itraconazol en el tratamiento de SAFS que por el momento no permiten recomendar su empleo rutinario (1). Los azoles actúan al disminuir la colonización fúngica en la vía área y por ende la respuesta inmune, posiblemente potenciando el efecto de los esteroides y por medio de efectos inmunológicos directos $(1,2)$.

El seguimiento del tratamiento se realiza a través de la medición de $\operatorname{IgE}$ total, radiografía de tórax, función pulmonar y medición de calidad de vida (2). Las complicaciones de ABPA incluyen exacerbaciones recurrentes, colapso de las vías aéreas por impactación de moco, bronquiectasias, aspergilosis broncopulmonar crónica, fibrosis, cor pulmonale y falla respiratoria crónica $(2,5)$.

En este artículo se reporta el caso de un paciente con asma severa con evidencia de sensibilización fúngica tanto por prick test como por IgE específica para Aspergillus. El valor de IgE total fue menor de $1.000 \mathrm{UI} / \mathrm{ml}$ y mediante escanografía se evidenciaron engrosamiento peribronquial y bronquiolectasias que podrían tratarse de una manifestación inicial del daño pulmonar debido a la hipersensibilidad a Aspergillus. Lo anterior configura un asma severa con sensibilización fúngica, pero en vista del nivel de IgE total posiblemente subestimado por el uso de esteroides y ante el deterioro clínico del paciente, persistió la duda diagnóstica de una aspergilosis broncopulmonar alérgica seropositiva y se inició de manejo médico.

\section{Conclusión}

Con el fin de minimizar complicaciones potencialmente irreversibles en pacientes con asma, se recomienda un gran índice de sospecha para ABPA y SAFS, y por tanto es conveniente plantear la búsqueda activa de sensibilización fúngica por medio de pruebas de hipersensibilidad cutánea. Infortunadamente, una vez se observan las manifestaciones radiológicas descritas, la enfermedad podrá estar muy avanzada y conllevar mal pronóstico. De ahí que la meta sea llegar a un diagnóstico temprano para así prevenir su progresión, y no solo mejorar la sobrevida de los pacientes sino minimizar el uso de recursos de salud.

\section{Conflictos de interés}

Los autores declaran no tener conflictos de interés.

\section{Bibliografía}

1. Agarwal R. Severe asthma with fungal sensitization. Curr Allergy Asthma Resp. 2011;11:403-13.

2. Agarwal R, Chakrabarti A, Shah A, Gupta D, et al. Allergic broncopulmonary aspergilosis: review of the literature and proposal of new diagnostic and classification criteria. Clin Exp Allerg. 2013;43:850-73.

3. Agarwal R, Aggarwal N, Gupta D, Jindal K. Aspergillus hypersensitivity and allergic bronchopulmonary aspergillosis in patients with bronchial asthma: systematic review and meta-analysis. Int J Tuberc Lung Dis. 2009;13:936-44.

4. Patterson K, Strek Mary E. Diagnosis and treatment of pulmonary aspergillosis syndromes. Chest. 2014;146 (5):135868.

5. Hogan C, Denning D. Allergic bronchopulmonary aspergillosis and related allergic syndromes. Sem Resp Crit Care Med. 2011;32(6):682- 92.

6. Agarwal R. Allergic bronchopulmonary aspergillosis. Chest. 2009;135:805-26.

7. Greenberg P. When to suspect and work up allergic bronchopulmonary aspergillosis. Ann Allergy Asthma Inmunol. 2013;111:1-4.

8. Agarwal R, Khan A, Garg M, Aggarwal AN, Gupta D. Chest radiographic and computed tomographic manifestations in allergic bronchopulmonary aspergillosis. World J Radiol. 2012;4:141-50.

9. Menzies D, Holmes L, McCumesky G, Prys-Picard C, Niven R. Aspergillus sensitization is associated with airflow limitation and bronchiectasis in severe asthma. Allergy. 2011;66:679-85.

10. Agarwal R, Hhan A, Gupta D, Aggarwal A, Saxena AK, Chakrabarti A. An alternate method of classifying allergic bronchopulmonary aspergillosis based on high-attenuation mucus. PLoS One. 2010;12:e15346.

11. Franquet T, Müller NL, Gimenez A, Gumbe P, de la Torre J, Bagué S. Spectrum of pulmonary aspergillosis: histolo- 
gic, clinical, and radiologic findings. RadioGraphics. 2001; 21:825-37.

12. Angus RM, Davies ML, Cowan MD, McSharry C, Thomson NC. Computed tomographic scanning of the lung in patients with allergic bronchopulmonary aspergillosis and in asthmatic patients with a positive skin test to Aspergillus fumigatus. Thorax. 1994;49:586-89.

13. Patterson R, Greenberg PA, Halwig JM, et al. Allergic bronchopulmonary aspergillosis: natural history and classification of early disease by serologic and roetgenographic. Arch Intern Med. 1986;146(5):916-18.

14. Greenberger PA. Allergic bronchopulmonary aspergillosis. J Allergy Clin Immunol. 2002;110: 685-92.
15. Mahdavinia M, Grammer LC. Management of allergic bronchopulmonary aspergillosis: a review and update. Ther Adv Respir Dis. 2012;6:173-87.

16. Hamilton Rg, Marcotte GV, Saini SS. Inmunological methods for quatifying free and total serum IgE levels in allergy patients receiving omalizumab (Xolair) therapy. J Immunol Methods. 2005;303(1-2):81-91.

17. Agarwal R, Khan A, Aggarwal AN, et al. Clinical relevance of peripheral blood eosinophil count in allergic bronchopulmonary apergillosis. J Infect Public Health. 2011;4:235-43.

18. Jat KR, Walia DK, Khairwa A. Anti-IgE therapy for allergic bronchopulmonary aspergillosis in people with cystic fibrosis. Cochrane Database Syst Rev. 2015;4:11. 